\title{
Quantification of internalization of EGFR-binding Affibody molecules: Methodological aspects
}

\author{
LOVISA GÖSTRING ${ }^{1,2}$, MING TSUEY CHEW ${ }^{1}$, ANNA ORLOVA ${ }^{1,2}$, INGMARIE HÖIDÉN-GUTHENBERG ${ }^{2}$, \\ ANDERS WENNBORG ${ }^{2}$, JÖRGEN CARLSSON ${ }^{1}$ and FREDRIK Y. FREJD ${ }^{1,2}$

\begin{abstract}
${ }^{1}$ Biomedical Radiation Sciences, Rudbeck Laboratory, Department of Oncology, Radiology and Clinical Immunology, Uppsala University, SE-751 85 Uppsala; ${ }^{2}$ Affibody AB, PO Box 20137, SE-161 02 Bromma, Sweden
\end{abstract}

Received August 11, 2009; Accepted October 5, 2009

DOI: 10.3892/ijo_00000551

\begin{abstract}
Tumor cell internalization of targeting agents is of interest, since internalization influences the local retention time of a radionuclide and thereby imaging quality in PET and SPECT and effects of radionuclide therapy. In cases where nuclear methods are not applicable at the cellular level, quantitative fluorescent techniques are useful as described in this article. Two fluorescence-based methods to study cellular internalization were applied: the CypHer and the Alexa488quenching methods, both utilized in fluorescence microscopy and flow cytometry. Two EGFR-binding Affibody molecules were analyzed in A431 cells: the monomer Z1907 and the dimer (Z1907) $)_{2}$. EGF, cetuximab and non-specific Affibody molecules were used as controls. For comparison, internalization of ${ }^{111}$ In-labeled Z1907 was studied with the acid wash internalization assay. The Cypher method is straightforward, but requires equal labeling of all compounds for accurate quantification. The Alexa488-quenching method is preferable since it is independent of the dye-to-protein ratio. According to this method, about $45 \%$ of EGF and $19-24 \%$ of the bound Affibody molecules and cetuximab were internalized within one hour. Similar results were seen with ${ }^{111} \mathrm{In}-\mathrm{Z} 1907$ in the acid wash method, while (Z1907) ${ }_{2}$ was not removed by acid and thus could not be studied this way. The fluorescence-based Alexa488-quenching method is well suited to quantitatively analyze internalization of targeting agents, also those that resist acid wash. The internalized fraction showed that both the monomeric and dimeric Affibody molecules are expected to give good uptake and thereby good retention of metallic radionuclides which will render good tumor to background values.
\end{abstract}

Correspondence to: Dr Lovisa Göstring, Biomedical Radiation Sciences, Department of Oncology, Radiology and Clinical Immunology, Rudbeck Laboratory, Uppsala University, SE-751 85 Uppsala, Sweden

E-mail: lovisa.gostring@bms.uu.se

Key words: ${ }^{111}$ In, A431, Affibody molecule, Alexa488, cetuximab, CypHer5E, EGFR, internalization, radionuclide, retention

\section{Introduction}

The epidermal growth factor receptor (EGFR) is an interesting target for radionuclide-based tumor imaging and therapy, since it is overexpressed in many human tumors such as head and neck, bladder, breast and lung cancers $(1,2)$. It is a member of the ErbB receptor tyrosine kinase family, which consists of EGFR (or HER1 or ErbB1), HER2/ErbB2, HER3/ErbB3 and HER4/ErbB4 (3). Ligand binding to EGFR induces dimerization with another EGFR or with other members of the ErbB family, and activates tyrosine kinase residues on the intracellular domains of the proteins (4). This recruits down-stream signaling proteins, triggering signal cascades along a number of pathways that eventually lead to cell growth, migration and apoptosis resistance $(5,6)$.

Upon ligand binding, EGFR is internalized into endosomes. The early endosomes and their receptor cargo are either recycled to the membrane (7), or further processed into late endosomes/multivesicular bodies and lysosomes, where the receptor and its ligand are degraded (8). Internalization is generally believed to occur via the classical clathrin-coated pit pathway, but internalization via non-clathrin pathways has been reported as well (9).

For nuclear medicine applications, the internalization degree of a radionuclide-delivering EGFR binding agent is of interest. Internalization will influence the local retention time of the radionuclide and thereby influence the imaging quality in PET and SPECT, as well as the radionuclide therapy effect. The classical way to study internalization is using the 'acid wash' method, where the surface-bound fraction of a radiolabeled agent is removed under acidic conditions and the remaining cell-bound fraction is considered to be internalized. However, this method has its limitations, since not all compounds are easily washed off with acid. In such cases, fluorescent methods to quantify internalization must be applied.

Recently, high-affinity Affibody molecules specific for EGFR and HER2 have been generated. Affibody molecules are three-helical proteins derived from the B domain of the IgG-binding staphylococcal protein A (10). Their small size $(7 \mathrm{kDa})$ and robust structure make them suitable for cancer imaging and therapy (11). A HER2-specific Affibody molecule has earlier been shown to render high-contrast radionuclide imaging in preclinical studies $(12,13)$, and in early clinical reports (14). Another candidate for molecular imaging, the 
EGFR-binding Affibody molecule Z1907, has also shown promising results in preclinical studies, both in terms of cell binding and retention and in terms of in vivo tumor targeting of grafted EGFR-expressing tumors $(15,16)$. So far however, it has not been known to what extent the Z1907 molecule is internalized.

In the present work, we have studied internalization of the monomeric (monovalent) Z1907 and the dimeric (divalent) (Z1907) 2 Affibody molecules on EGFR-expressing A431 cells. We wanted to know whether the monomer and dimer differed in internalization, and to see if there is a method suitable for internalization screening of targeting agents. Two different internalization methods were tested: labeling of the Affibody molecules with the CypHer fluorophore, and quenching of Alexa488-labeled Affibody molecules with an anti-Alexa488 antibody. CypHer is a fluorophore that is about 10 times more fluorescent at low pH, e.g. in acidic intracellular vesicles such as endosomes an lysosomes, than at neutral pH (17). By labeling proteins with this fluorophore one can easily study their internalization in a microscope or flow cytometer $(18,19)$. The other method was adopted from Austin et al (20). In this method, the cells are incubated with Alexa488-labeled Affibody molecules, and addition of an anti-Alexa488 antibody quenches fluorescence on the cell surface but not from internalized molecules (21). In this way, a ratio between internalized molecules and total amount of bound molecules can be calculated. The natural ligand EGF and the humanized anti-EGFR antibody cetuximab were used as reference substances. EGF is known to trigger internalization and degradation of EGFR (22), and cetuximab is also internalized when bound to EGFR although this process is less studied $(2,23)$.

\section{Materials and methods}

Cell culture. The epidermal squamous carcinoma A431 cells (ECACC 85090402) were cultured in Earle's MEM with glutamine (Cambrex) supplemented with $10 \%$ fetal calf serum (Sigma), non-essential amino acids (Gibco) and PEST (penicillin $100 \mathrm{IU} / \mathrm{ml}$, streptomycin $100 \mu \mathrm{g} / \mathrm{ml}$ ). These cells were used at our laboratory in Bromma where all Alexa488 experiments were carried out. The immunofluorescence stainings with CypHer and the acid wash experiments were performed at our laboratory in Uppsala, where A431 cells from ATCC (CRL 1555) were used. These were cultured in Ham's F10 medium supplemented with $10 \%$ fetal calf serum, $2 \mathrm{mM}$ L-glutamine and PEST (penicillin $100 \mathrm{IU} / \mathrm{ml}$, streptomycin $100 \mu \mathrm{g} / \mathrm{ml}$ ).

Chemicals and reagents. Recombinant EGF was purchased from Chemicon. Monomeric (Z1907) and dimeric (Z1907) anti-EGFR Affibody ${ }^{\circledR}$ molecules as well as the negative controls, the amyloid beta binding $(\mathrm{ZA} \beta)_{2}(24)$ and the Taq polymerase-binding ZTaq (25) Affibody molecules were produced by Affibody AB (Bromma, Sweden). These have previously been used as in vitro and in vivo controls (26-29). The chimeric monoclonal anti-EGFR antibody cetuximab (Erbitux) was purchased from Apoteket AB, Sweden.

CypHer labeling of EGFR-binding agents. EGF, Z1907, $(\mathrm{Z} 1907)_{2}$, cetuximab and $(\mathrm{ZAB})_{2}$ were labeled with the $\mathrm{pH}$ - dependent fluorophore CypHer ${ }^{\mathrm{TM}}$ 5E mono NHS ester (Amersham Biosciences, GE Healthcare) in accordance with the manufacturer's instructions. CypHer ${ }^{\mathrm{TM}}$ 5E mono NHS diluted in DMSO was mixed with the respective proteins diluted in PBS and sodium carbonate $0.5 \mathrm{M}, \mathrm{pH}$ 8.3. This mixture, with a molar ratio of CypHer5E to protein of 5:1, was incubated at room temperature for $1 \mathrm{~h}$ in the dark. Uncoupled CypHer5E was removed by dialysis in Slide-ALyzers (Pierce) in PBS at $4{ }^{\circ} \mathrm{C}$ overnight. Concentration and degree of labeling were calculated from absorption measurements with a spectrophotometer (NanoDrop ND-1000).

Alexa488 labeling of EGFR-binding reagents. EGF, Z1907, $(\mathrm{Z} 1907)_{2}$, cetuximab and $(\mathrm{ZAB})_{2}$ were labeled with Alexa Fluor 488 carboxylic acid, succinimidyl ester (Molecular Probes) according to the manufacturer's instructions. One millgram of each EGFR-binding agent was labeled with 5 times molar excess of Alexa488 resuspended in DMSO. The mixture was incubated for $1 \mathrm{~h}$ at room temperature, and free Alexa488 was removed in 'Zeba desalt Spin Columns', $5 \mathrm{ml}$ (Pierce). Concentration and degree of labeling were calculated from absorption measurements done with a spectrophotometer (NanoDrop ND-1000).

Labeling of Bz-DTPA-Z1907 with ${ }^{111}$ In. Labeling was done as previously reported (30). In short, $25 \mu \mathrm{g}$ of Bz-DTPA-Z1907 was mixed with $34 \mu$ l stock solution of ${ }^{111} \mathrm{InCl}(25 \mathrm{MBq}$, Mallinckrodt, The Netherlands) followed by $35 \mu 11 \mathrm{M}$ ammonium acetate buffer $(\mathrm{pH} \sim 5.0)$. The reaction mixture was incubated at $60^{\circ} \mathrm{C}$ for $1 \mathrm{~h}$ and labeling yield was checked on ITLC plates (Gelman Sciences Inc.) eluted with $0.2 \mathrm{M}$ citric acid (p.a. Merck). ITLC plates were developed on Phosphor Imager (Cyclone ${ }^{\mathrm{TM}}$ Storage Phosphor System with OptiQuant $^{\mathrm{TM}}$ Image analysis System).

Confocal microscopy with CypHer5E-labeled agents. Internalization of CypHer5E-labelled EGF, Z1907, (Z1907) cetuximab and $(\mathrm{ZA} \beta)_{2}$ was tested on A431 cells. Cells $\left(2.3 \times 10^{4}\right)$ were seeded on cover glasses and allowed to grow in complete Ham's F10 medium for $40 \mathrm{~h}$. The glasses were washed in serum-free medium and incubated in $100 \mu \mathrm{l}$ serum-free medium containing $0.3 \mu \mathrm{M}$ CypHer5E-labeled protein, and $2 \mu \mathrm{M}$ Hoechst 33342 (Sigma Fluka) for $1.5 \mathrm{~h}$ at $37^{\circ} \mathrm{C}$. The glasses were then washed in serum-free medium, and photographs were taken in a live-chamber cooled to $4^{\circ} \mathrm{C}$ in a Zeiss LSM 510 META confocal microscope (objective: PlanApochromat 63x/1.4 Oil DIC).

Flow cytometry with CypHer5E-labeled agents. Cells were stained in a multi-well plate but transferred to tubes before analyzed in a flow cytometer. A431 cells were trypsinized, neutralized and suspended in serum-free EMEM medium. Cells $\left(3 \times 10^{5}\right)$ were incubated with CypHer5E-labeled EGFR binders of various concentrations for $1 \mathrm{~h}$ at $37^{\circ} \mathrm{C}$, washed twice and resuspended in PBS and analyzed in a flow cytometer (FACS Canto II, BD Biosciences). The mean fluorescence of each reading was calculated by the software (FACS Diva, BD Biosciences). In order to make the results from five different experiments compatible, the fluorescence intensity of each experiment was normalized. For each experiment, the 
mean fluorescence of each compound and concentration was divided by the mean fluorescence of unstained cells from that reading. Mean and standard error of the corresponding values from the five different experiments were calculated.

Quenching of Alexa488 by use of the anti-Alexa488 antibody microscopy. A431 cells $\left(1 \times 10^{4}\right)$ were seeded in complete EMEM medium on 8-well slides (Histolab) and allowed to grow for $24 \mathrm{~h}$. They were then incubated with $100 \mathrm{nM}$ of Alexa488-labelled EGF, Z1907, $(\mathrm{Z1907})_{2}$, cetuximab or ZTaq in complete medium for $1 \mathrm{~h}$ at $37^{\circ} \mathrm{C}$. The cells were gently washed in cold PBS and incubated in PBS (control) or $500 \mathrm{nM}$ quenching anti-Alexa488 antibodies (Molecular Probes) for $1 \mathrm{~h}$ on ice. After fixation in $2 \%$ formaldehyde, the slides were washed in PBS and mounted in anti-fading containing DAPI (Vectahield). Pictures were taken in a Leica microscope (DMLA).

Quenching of Alexa488 by use of the anti-Alexa488 antibody, flow cytometry and calculation of internalized fraction of EGFR binders. Two somewhat different protocols for this method were used, 'version 1' and 'version 2'. Version 1: $50 \mathrm{nM}$ of Alexa488-labelled EGF, Z1907, (Z1907) $)_{2}$ or cetuximab were incubated with $3 \times 10^{5}$ A431 cells (ECACC) in complete medium for $1 \mathrm{~h}$ at 37 or $0^{\circ} \mathrm{C}$, respectively. The cells were washed twice in ice cold PBS in a cold centrifuge, and resuspended in $500 \mathrm{nM}$ quenching anti-Alexa488 antibody diluted in ice cold PBS. Identical control tubes were incubated in PBS only. All tubes were incubated for $1 \mathrm{~h}$ on ice. Without washing, all cells were fixed in two volumes of $2 \%$ paraformaldehyde for $10 \mathrm{~min}$ at room temperature. The paraformaldehyde was removed by one wash in PBS, and the cells were resuspended in PBS and analyzed in a flow cytometer (FACS Canto II, BD Biosciences). Mean fluorescence from the flow cytometry reading of $2 \times 10^{4}$ cells per tube was used for the calculations. Internalization was calculated as fluorescence of quenched cells (intracellular compartments only) divided by that of unquenched cells (both cell surface and intracellular compartments). The cells incubated with Alexa488-labelled compounds on ice were used as a control where the antibody's quenching efficiency on each Alexa-labeled compound was estimated. Since nothing should be internalized at $0^{\circ} \mathrm{C}$, the fluorescence measured after quenching with anti-Alexa on these cells was considered to be unquenchable surface fluorescence (background). This amount (5-15\% of total fluorescence) was corrected for when the percentage internalized compound was calculated. Version 2: In a variant of the method above, the cells were preincubated with the same binders for $1 \mathrm{~h}$ on ice to allow binding to the EGFR receptors but not internalization. The cells were washed in ice-cold PBS to remove unbound EGFR binders, incubated for $1 \mathrm{~h}$ at $37^{\circ} \mathrm{C}$ and further treated as described above. In this way, there was no constant presence of Alexa488-labelled binders but only the ones bound to the receptors were studied. This method was adopted from Austin et al (20).

Acid wash with ${ }^{111}$ In-labeled Z1907. This method was performed as described earlier (31). In short, A431 cells (ATCC) were seeded in petri dishes, $5 \times 10^{5}$ cells per dish, and allowed to grow in complete medium overnight. The cells were then
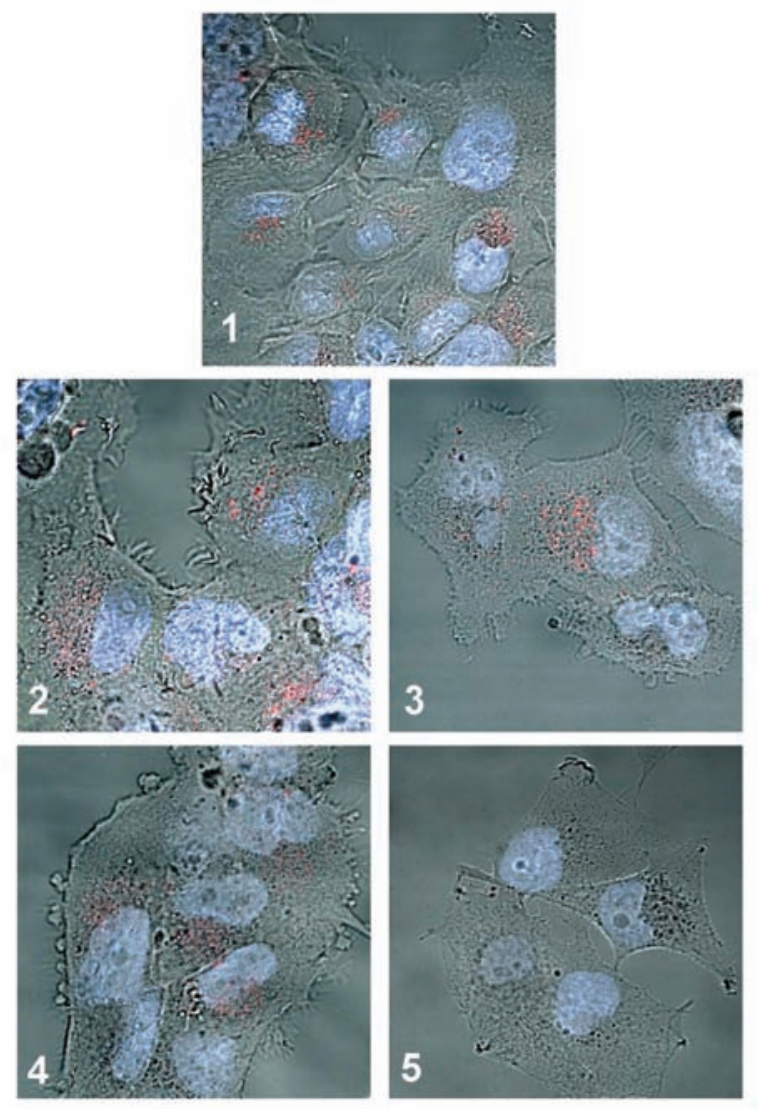

Figure 1. A431 cells stained with CypHer5E-labeled EGFR binding compounds (red). The nuclei are counterstained with Hoechst (blue). (1) EGF, (2) Z1907, (3) $(\mathrm{Z} 1907)_{2}$, (4) cetuximab and (5) (ZAß) 2 (negative control). Overlay of confocal fluorescence and phase contrast photomicroscopy.

washed and incubated with $6.5 \mathrm{nM}$ (corresponding to $5 \mathrm{x}$ molar excess to the receptors) ${ }^{111}$ In-labelled Z1907 in complete medium at $37^{\circ} \mathrm{C}$ for $0-60 \mathrm{~min}$. After washing in serum free medium, the cells were incubated with ice cold acid $(0.2 \mathrm{M}$ glycin, $0.15 \mathrm{M} \mathrm{NaCl}, 4 \mathrm{M}$ urea, $\mathrm{pH} 2.0$ ) on ice for $5 \mathrm{~min}$. The acid (with the cell surface fraction of Z1907) was collected and cells were washed with additional $0.5 \mathrm{ml}$ acidic solution. The cells were treated with $1 \mathrm{M} \mathrm{NaOH}$ and removed from the petri dish using a cell scraper. This cell suspension was kept as the internalized fraction of ${ }^{111} \mathrm{In}-\mathrm{Z} 1907$. For each time point, three petri dishes were used. Radioactivity was measured in a gamma counter (Wizard 1480). Internalization at each timepoint was calculated as $\mathrm{CPM}$ of the $\mathrm{NaOH}$ fraction divided by $\mathrm{CPM}$ of the acid $+\mathrm{NaOH}$ fractions.

\section{Results}

Confocal microscopy with CypHer-labeled agents. To confirm that the CypHer fluorophore was fluorescent only when internalized, A431 cells were stained with the CypHerlabeled agents and viewed in the fluorescence microscope (Fig. 1). As expected, the CypHer fluorescence could only be detected intracellularly and not on the cell surface. Both Z1907 and $(Z 1907)_{2}$ could be seen in small intracellular vesicles, a staining pattern very similar to that of EGF. Cetuximab showed a similar pattern but with somewhat smaller vesicular structures. The negative control $(\mathrm{ZA} B)_{2}$ showed no staining. 


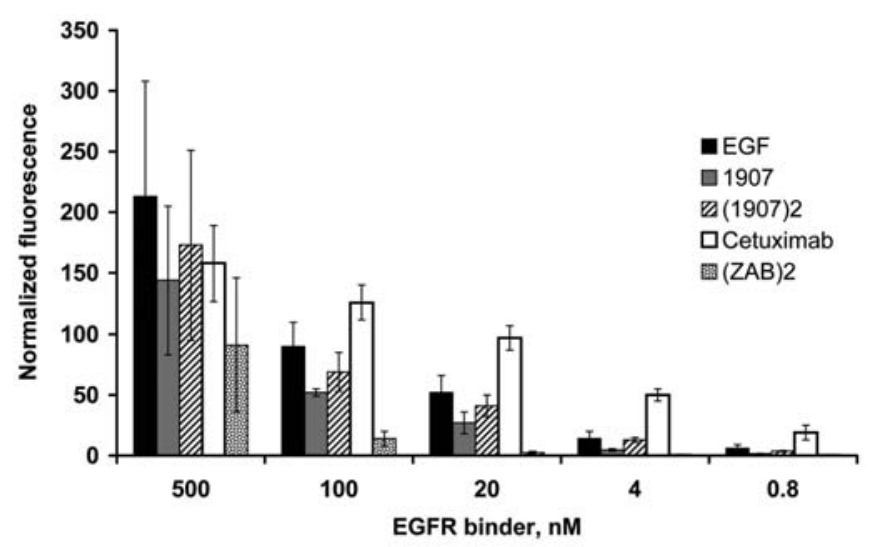

Figure 2. CypHer fluorescence intensity as a function of the amount of added CypHer5E-labeled EGFR binding compounds and the negative control $(\mathrm{ZA} ß)_{2}$. Studies were performed using flow cytometry. Results from five experiments are shown as mean values and standard errors.

Quantification of internalization using CypHer and flow cytometry analysis. Since CypHer becomes fluorescent when internalized, internalization of a CypHer-labeled compound can be easily measured in a flow cytometer. In this way, the four EGFR-binding compounds and the non-specific $(\mathrm{ZAB})_{2}$ were tested at different concentrations (Fig. 2). Both Z1907 and $(\mathrm{Z1907})_{2}$ were internalized, with no significant difference in signal between the two compounds. EGF seemed to have somewhat stronger signals, whereas the strongest signals were, in most cases, seen with cetuximab. According to the spectrophotometer analyses of the CypHer-labeled compounds, all agents except cetuximab had a dye-to-protein ratio of about 2. Cetuximab, which is a larger protein, had a dye-to-protein ratio of 3.2. No CypHer signal was seen from $(\mathrm{ZA} \beta)_{2}$ at lower concentrations, but at $500 \mathrm{nM}$ this non-specific signal was strong. For further studies, $50 \mathrm{nM}$ was chosen as the optimal concentration, as this concentration would render strong specific signals without visible unspecific uptake.

Specificity test with flow cytometry analysis. In order to confirm that the CypHer signal was specific, the A431 cells were preincubated with 100 -fold molar excess of unlabeled compound before incubated with the corresponding CypHerlabeled compound. The CypHer signal of all four substances was blocked to levels similar to that of the non-specific substance $(\mathrm{ZA} \beta)_{2}$ (data not shown).

Quenching of surface fluorescence using the anti-Alexa488 antibody, microscopy. A431 cells grown on glass slides were incubated with Alexa488-labeled EGFR binders for $1 \mathrm{~h}$, washed and incubated on ice with or without the fluorescencequenching anti-Alexa antibody. As seen in Fig. 3, most surface fluorescence was quenched by the antibody and mainly intracellular fluorescence remained. When cells stained with Alexa488-labeled Z1907, (Z1907) 2 or cetuximab were incubated with PBS only ('unquenched'), most fluorescence was located to the cell membrane. However, with unquenched Alexa488-EGF the membrane staining was less pronounced and the majority of the fluorescence was located to intracellular compartments. Only faint fluorescence was seen with the control substance ZTaq.

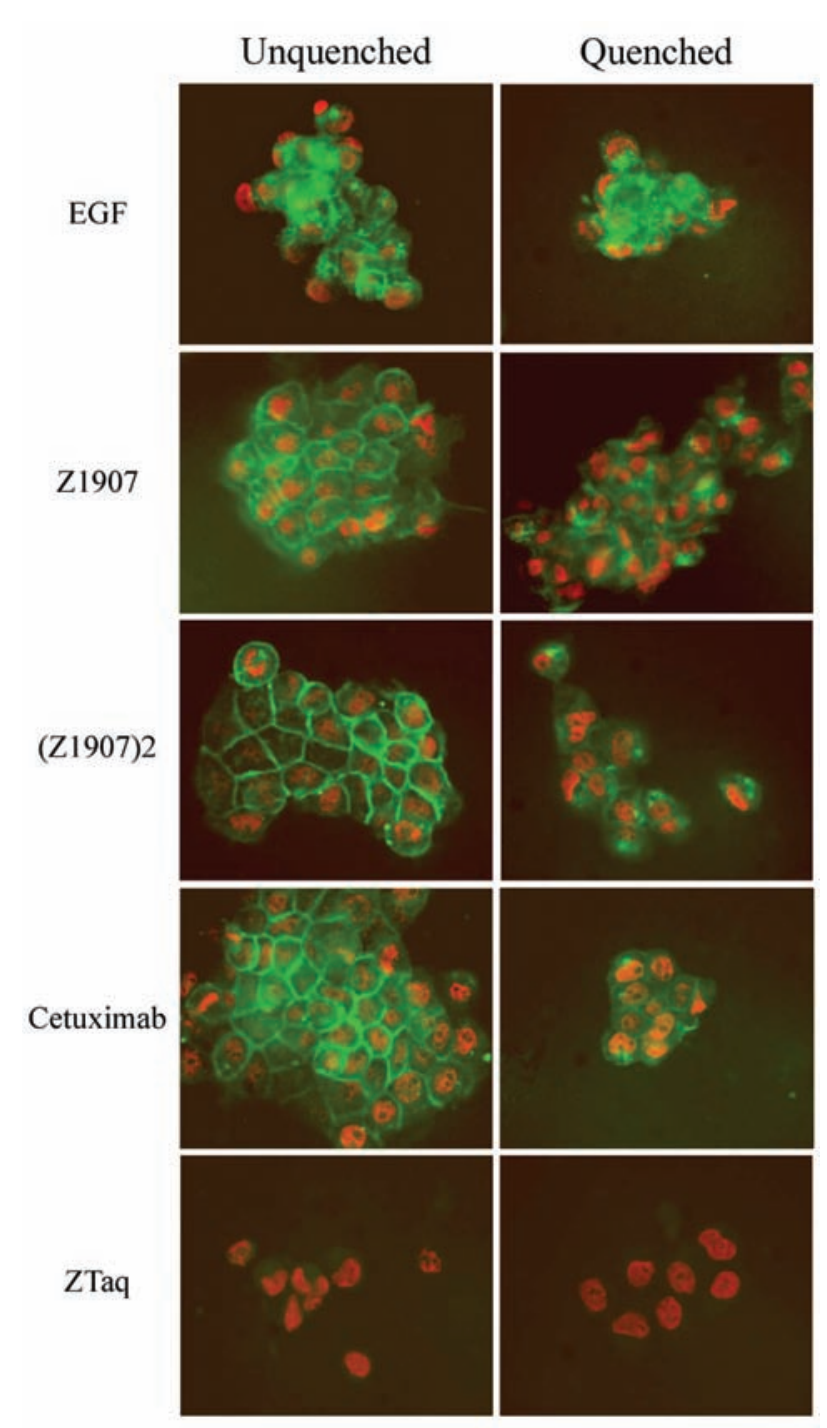

Figure 3. Quenching of surface-bound fluorescence from Alexa488-labeled EGFR binders. Unquenched control cells (left column) and cells where surface fluorescence is quenched with an anti-Alexa488 antibody (right column).

Quenching of surface fluorescence using the anti-Alexa488 antibody, determination of internalized fraction using flow cytometry. Internalization was measured as percent of total amount bound EGFR binder by comparing Alexa488 fluorescence of quenched cells to that of unquenched ones in flow cytometer analysis. Two versions of this method were performed, version 1 where the fluorescent binders were constantly present during internalization, and version 2 where the fluorescent binders were prebound to the cells on ice and the excess washed off before internalization was monitored at $37^{\circ} \mathrm{C}$. In both versions, unquenchable surface fluorescence (background) was estimated in parallel tubes on ice. This unquenchable amount was about 10-15\% with Alexa488EGF, and 5-10\% with Alexa488-labelled Z1907, (Z1907) ${ }_{2}$ and cetuximab. This 'background fraction' was corrected for in each individual experiment before the internalization percentage was calculated.

After $1 \mathrm{~h}$ at $37^{\circ} \mathrm{C}$, about $47 \%$ (version 1) or $41 \%$ (version 2) of the total cell fluorescence from Alexa488-EGF was intracellular (Fig. 4). With Z1907, (Z1907) ${ }_{2}$ and cetuximab this 
A

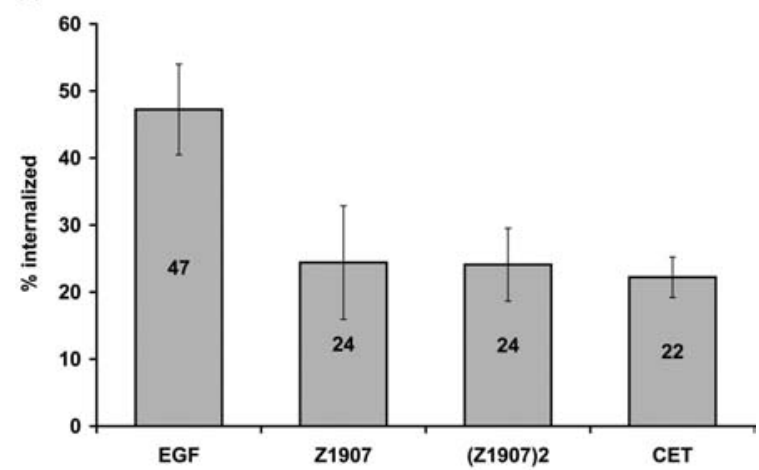

B

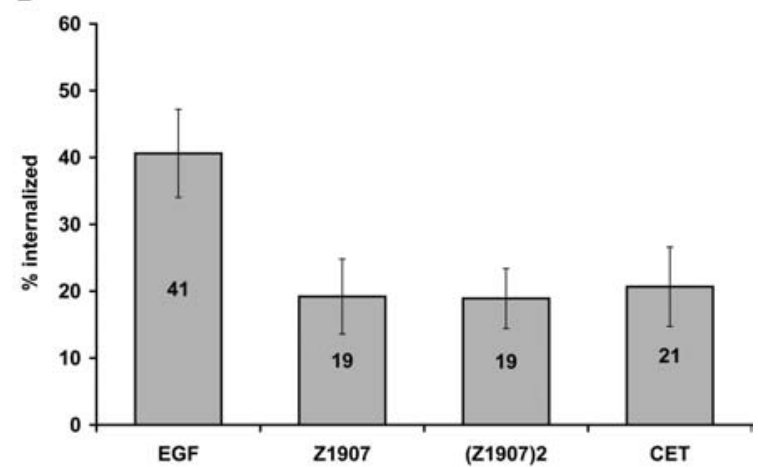

Figure 4. Internalization of Alexa488-labeled EGFR binders using the two versions of the Alexa488-quenching method. Cells were incubated in constant presence of the EGFR binders (A, version 1) or after pre-incubation on ice and removal of excess substance (B, version 2). Flow cytometry was used to estimate internalized fluorescence as percentage of total fluorescence. Mean values and standard deviations from 8 (panel A) and 9 (panel B) experiments are shown.

A

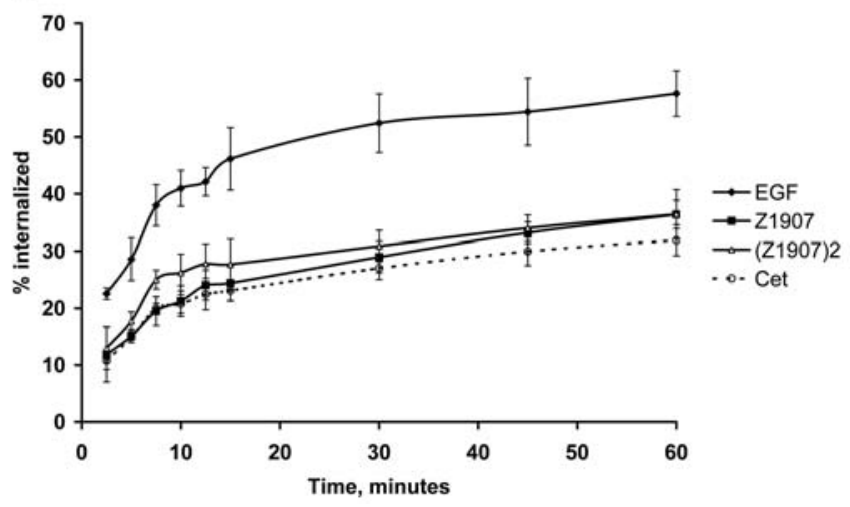

B

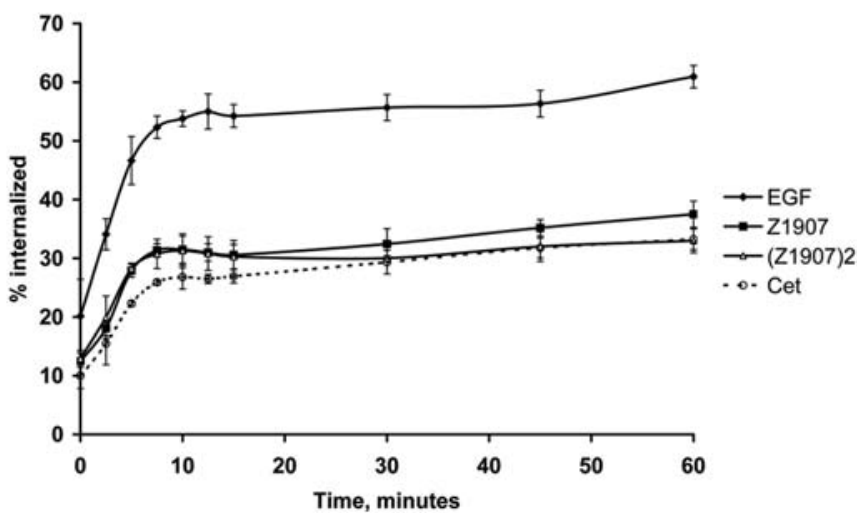

Figure 5. Internalization kinetics of Alexa488-labeled EGFR binders using the two versions of the Alexa488-quenching method. Cells were incubated in constant presence of the EGFR binders (A, version 1) or after pre-incubation on ice and removal of excess substance (B, version 2). Flow cytometry was used to estimate internalized fluorescence as percentage of total fluorescence. Mean values and standard deviations from 5 (A) and 3 (B) experiments are shown.

number was lower, 22-24\% with version 1 and 19-21\% with version 2 . There was no significant difference between these three binders. The results from the different method versions seemed fairly equal.

Study of internalization kinetics using the Alexa488 quenching antibody. The two versions of the Alexa488 method were also used for studies of internalization kinetics of the four compounds (Fig. 5). The end result after 60 min was similar with the two versions, when about $60 \%$ of EGF and $35 \%$ of the other binders were internalized. However, since the complex setup in the kinetics studies did not allow parallel studies on ice, the unquenchable surface fluorescence was not subtracted in this case. This is why the internalized fraction was about 10-20 percentage units higher after $60 \mathrm{~min}$ in the kinetics studies than in the results seen in Fig. 4. The internalization rate was faster with version 2 (Fig. 5B) than with version 1 (Fig. 5A), and was almost stabilized after about 10 min whereas in version 1 , internalization slowly increased throughout the incubation time. EGF had a significantly higher internalization rate than the other compounds. No major differences could be seen between Z1907, (Z1907) ${ }_{2}$ and cetuximab.

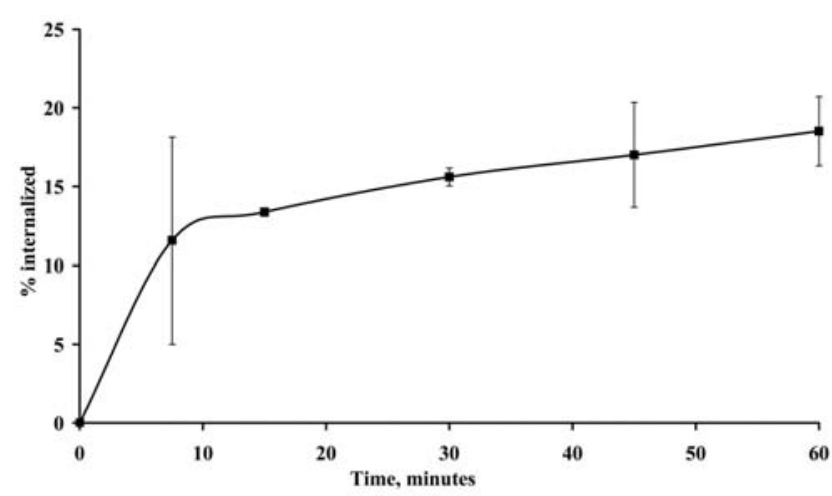

Figure 6. Internalization of ${ }^{111}$ In-Z1907 by A431 cells as a function of time during $60 \mathrm{~min}$. Each point corresponds to the mean value and standard deviation from measurements on three culture dishes.

Acid wash with ${ }^{111}$ In-labelled Z1907. In order to compare the results obtained with fluorochrome-based methods to a more commonly used technique, the monomeric affibody Z1907 was labeled with ${ }^{111} \mathrm{In}$ and used in an acid wash internalization study. The uptake of the radiolabeled affibody molecule was monitored for $60 \mathrm{~min}$ (Fig. 6). After $1 \mathrm{~h}$, about $19 \%$ of 
the cell associated radioactivity was intracellular. The dimeric (Z1907) 2 could not be analyzed with the acid wash method since it was not effectively washed away from the cellular membrane at low $\mathrm{pH}$ (not shown).

\section{Discussion}

When potential molecular candidates for tumor imaging and/or therapy are characterized, quantitative determination of cellular internalization is of importance. A toxin or radionuclide conjugated to a receptor-specific affinity protein may work more efficiently inside the cell than when localized on the cell surface, and longer retention time due to internalization will improve SPECT and PET images. Here we have studied internalization of two EGFR-binding Affibody molecules: Z1907 (monovalent) and (Z1907) ${ }_{2}$ (bivalent). EGF and cetuximab were used as reference substances and the nonEGFR binding Affibody molecules ZTaq and (ZAß) 2 as negative controls.

Two different fluorescence-based methods were used. The first method, CypHer labeling, measures fluorescence of the internalized molecule pool, while the second method, Alexa488-quenching, is based on the difference between total cell-bound and internalized fluorescence. In addition, the results were compared to results from ${ }^{111}$ In delivered with the monomer Z1907, using the acid wash internalization method. This method has been verified to be a reliable method for studies of internalization of EGF, and is a common way to study internalization. However, it has its limitations and was not applicable to the dimeric Affibody molecule, apparently due to acid-resistant binding to the cell membrane. Z1907 and (Z1907) 2 have earlier been studied at our laboratories using the so called ${ }^{111} \mathrm{In} /{ }^{125} \mathrm{I}$ method, which compares retention of the ${ }^{111} \mathrm{In}$-labeled binder (residualizing nuclide) to that of the ${ }^{125}$ I-labeled binder (unresidualizing nuclide) (16). This method estimates the influence of internalization and degradation on cellular retention, but is not a quantitative method to measure internalization.

Both fluorescent methods were visualized in immunofluorescence stainings (Figs. 1 and 3) and shown to be functional for the EGFR-binding and control substances used here. According to flow cytometer analyses of the CypHerlabeled EGFR-binders, Z1907 and (Z1907) $)_{2}$ were both internalized and no significant difference in signal could be seen between the two binders. EGF seemed to render somewhat stronger signals, whereas the strongest signals were seen with cetuximab. This may to some extent be a result of the higher dye-to-protein ratio, $\approx 3.2$, for cetuximab compared to the other compounds where the values are around 2.0. The NHS labeling chemistry couples the CypHer fluorophore to any lysine in the protein, which is probably why the large antibody $(150 \mathrm{kDa})$ has more fluorophores coupled to it than the other compounds (6-13 kDa). Thus, to compare two different compounds using this method, one must make sure they have equal dye-to-protein ratios.

Two versions of the Alexa488-quenching method described by Austin et al (20) were used: Version 1, where an excess of the Alexa488-labelled EGFR-binding compound was present throughout the $37^{\circ} \mathrm{C}$ incubation, and version 2, where the cells were pre-incubated with the labeled EGFR binders on ice and the excess removed before the incubation. In both versions, a surface fluorescence-quenching anti-Alexa488 antibody was used to distinguish surface fluorescence from internalized fluorescence, and an internalized-to-total fluorescence ratio was calculated for each compound.

The two versions of the quenching method showed nearly equal results after $1 \mathrm{~h}$, with somewhat less internalization seen with version 2 . A more pronounced difference was seen when internalization kinetics was studied. As expected, the initial internalization was faster when version 2 was applied, since the fluorescent molecules were already bound to the cell surface when the incubation time started. Both versions of this method may, however, be relevant depending on the goal of the study.

According to both versions of the quenching method, about $40-50 \%$ of the cell-associated EGF and $20-25 \%$ of the other compounds [Z1907, (Z1907) ${ }_{2}$ and cetuximab] had been internalized after $1 \mathrm{~h}$. This is in fairly good agreement with earlier acid wash studies on cetuximab internalization, where about $25-30 \%$ of the bound antibodies were internalized after $1 \mathrm{~h}$ (32). Although both EGF and cetuximab are known to be internalized, the dynamics and degree of EGFR downregulation seem to differ (32). Cetuximab is internalized more slowly than EGF, and is recycled more efficiently (23). From the results seen here, it is likely that the Affibody molecules are similar to the antibody in this respect. Z1907 and its unmaturated form Z955 bind to the same domain as cetuximab, i.e. domain III of the extracellular part of EGFR (18). Thus, the effect of the Affibody molecules on EGFR is probably more similar to that of cetuximab than to EGF, which activates the receptor and its internalization by simultaneous binding to domains I and III. Although the internalized amount of Affibody molecules was lower than that seen with the natural ligand EGF, it is probably enough to be useful for imaging and therapy when applying radiometals.

The monovalent Z1907, which according to preclinical studies is more suitable for in vivo imaging than the bivalent form (16), was radiolabeled with ${ }^{111}$ In and tested in an acid wash internalizaion assay. With this assay, about $19 \%$ of bound ${ }^{111}$ In delivered by Z1907 were internalized after $1 \mathrm{~h}$. This is in accordance with the results from the surface quenching method. Delivery of ${ }^{111}$ In with (Z1907) $)_{2}$ was also studied using the acid wash method, but this bivalent Affibody molecule could not be washed away with the acid, although various protocols were tested (data not shown). This is a drawback of the acid wash method, where differences in $\mathrm{pH}$ sensitivity must be taken into account. For instance, cetuximab is not removed as readily as EGF by acid wash (23).

In summary, for quantitative internalization screenings of targeting agents, the Alexa488 quenching method is useful. The CypHer method requires the same dye-to-protein ratio for all compounds to be compared, while the Alexa488 quenching method instead compares each compound to itself, regardless of the dye-to-protein ratio, and this is an advantage. Acid wash is a well-established method for EGF but is not useful for all binders, since some are more resistant to acid-induced detachment from the membrane. Thus, the fluorescence-based Alexa488 quenching method may be a necessary complement to the more commonly used radioactivity-based acid wash method. 


\section{Acknowledgements}

This study was funded by Grant P25882-1 from the Swedish Governmental Agency for Innovation Systems (VINNOVA) and Grant 0980-B07-20XCC from the Swedish Cancer Society.

\section{References}

1. Salomon DS, Brandt R, Ciardiello F and Normanno N: Epidermal growth factor-related peptides and their receptors in human malignancies. Crit Rev Oncol Hematol 19: 183-232, 1995.

2. Vincenzi B, Schiavon G, Silletta M, Santini D and Tonini G: The biological properties of cetuximab. Crit Rev Oncol Hematol 68: 93-106, 2008.

3. Burden S and Yarden Y: Neuregulins and their receptors: a versatile signaling module in organogenesis and oncogenesis. Neuron 18: 847-855, 1997.

4. Ushiro $\mathrm{H}$ and Cohen $\mathrm{S}$ : Identification of phosphotyrosine as a product of epidermal growth factor-activated protein kinase in A-431 cell membranes. J Biol Chem 255: 8363-8365, 1980.

5. Yarden $Y$ and Sliwkowski MX: Untangling the ErbB signalling network. Nat Rev Mol Cell Biol 2: 127-137, 2001.

6. Scaltriti $\mathrm{M}$ and Baselga $\mathrm{J}$ : The epidermal growth factor receptor pathway: a model for targeted therapy. Clin Cancer Res 12: 5268-5272, 2006.

7. Sorkin A, Krolenko S, Kudrjavtceva N, Lazebnik J, Teslenko L, Soderquist AM and Nikolsky N: Recycling of epidermal growth factor-receptor complexes in A431 cells: identification of dual pathways. J Cell Biol 112: 55-63, 1991.

8. Carpentier JL, White MF, Orci L and Kahn RC: Direct visualization of the phosphorylated epidermal growth factor receptor during its internalization in A-431 cells. J Cell Biol 105: 2751-2762, 1987.

9. Sigismund S, Woelk T, Puri C, Maspero E, Tacchetti C, Transidico P, Di Fiore PP and Polo S: Clathrin-independent endocytosis of ubiquitinated cargos. Proc Natl Acad Sci USA 102: 2760-2765, 2005

10. Nord K, Gunneriusson E, Ringdahl J, Stahl S, Uhlen M and Nygren PA: Binding proteins selected from combinatorial libraries of an alpha-helical bacterial receptor domain. Nat Biotechnol 15: 772-777, 1997.

11. Nilsson FY and Tolmachev V: Affibody molecules: new protein domains for molecular imaging and targeted tumor therapy. Curr Opin Drug Discov Devel 10: 167-175, 2007.

12. Orlova A, Magnusson M, Eriksson TL, Nilsson M, Larsson B, Hoiden-Guthenberg I, Widstrom C, Carlsson J, Tolmachev V, Stahl S and Nilsson FY: Tumor imaging using a picomolar affinity HER2 binding affibody molecule. Cancer Res 66: 4339-4348, 2006.

13. Ekblad T, Tran T, Orlova A, Widstrom C, Feldwisch J Abrahmsen L, Wennborg A, Karlstrom AE and Tolmachev V: Development and preclinical characterisation of $(99 \mathrm{~m}) \mathrm{Tc}$ labelled Affibody molecules with reduced renal uptake. Eur J Nucl Med Mol Imaging 35: 2245-2255, 2008.

14. Baum R, Orlova A, Tolmachev V and Feldwisch J: A novel molecular imaging agent for diagnosis of recurrent HER2 positive breast cancer. First time in human study using an indium-111or Gallium-68-labeled Affibody molecule. Eur J Nucl Med Mol Imaging 33: S91, 2006.

15. Friedman M, Orlova A, Johansson E, Eriksson TL, HoidenGuthenberg I, Tolmachev V, Nilsson FY and Stahl S: Directed evolution to low nanomolar affinity of a tumor-targeting epidermal growth factor receptor-binding affibody molecule. J Mol Biol 376: 1388-1402, 2008.

16. Tolmachev V, Friedman M, Sandstrom M, Eriksson TL, Rosik D, Hodik M, Stahl S, Frejd FY and Orlova A: Affibody molecules for epidermal growth factor receptor targeting in vivo: aspects of dimerization and labeling chemistry. J Nucl Med 50: 274-283, 2009
17. Beletskii A, Cooper M, Sriraman P, Chiriac C, Zhao L, Abbot S and $\mathrm{Yu}$ L: High-throughput phagocytosis assay utilizing a $\mathrm{pH}$ sensitive fluorescent dye. Biotechniques 39: 894-897, 2005.

18. Nordberg E, Friedman M, Gostring L, Adams GP, Brismar H, Nilsson FY, Stahl S, Glimelius B and Carlsson J: Cellular studies of binding, internalization and retention of a radiolabeled EGFR-binding affibody molecule. Nucl Med Biol 34: 609-618, 2007.

19. Steffen AC, Gostring L, Tolmachev V, Palm S, Stenerlow B and Carlsson $\mathrm{J}$ : Differences in radiosensitivity between three HER2 overexpressing cell lines. Eur J Nucl Med Mol Imaging 35: 1179-1191, 2008.

20. Austin CD, De Maziere AM, Pisacane PI, van Dijk SM, Eigenbrot C, Sliwkowski MX, Klumperman J and Scheller RH: Endocytosis and sorting of ErbB2 and the site of action of cancer therapeutics trastuzumab and geldanamycin. Mol Biol Cell 15: 5268-5282, 2004.

21. Sklar LA, Oades ZG, Jesaitis AJ, Painter RG and Cochrane CG: Fluoresceinated chemotactic peptide and high-affinity antifluorescein antibody as a probe of the temporal characteristics of neutrophil stimulation. Proc Natl Acad Sci USA 78: 7540-7544, 1981.

22. Sorkin A and Goh LK: Endocytosis and intracellular trafficking of ErbBs. Exp Cell Res 315: 683-696, 2009.

23. Jaramillo ML, Leon Z, Grothe S, Paul-Roc B, Abulrob A and O'Connor McCourt M: Effect of the anti-receptor ligandblocking 225 monoclonal antibody on EGF receptor endocytosis and sorting. Exp Cell Res 312: 2778-2790, 2006.

24. Gronwall C, Jonsson A, Lindstrom S, Gunneriusson E, Stahl S and Herne N: Selection and characterization of Affibody ligands binding to Alzheimer amyloid beta peptides. J Biotechnol 128: 162-183, 2007.

25. Gunneriusson E, Nord K, Uhlen M and Nygren P: Affinity maturation of a Taq DNA polymerase specific affibody by helix shuffling. Protein Eng 12: 873-878, 1999.

26. Friedman M, Nordberg E, Hoiden-Guthenberg I, Brismar H, Adams GP, Nilsson FY, Carlsson J and Stahl S: Phage display selection of Affibody molecules with specific binding to the extracellular domain of the epidermal growth factor receptor. Protein Eng Des Sel 20: 189-199, 2007.

27. Renberg B, Shiroyama I, Engfeldt T, Nygren PK and Karlstrom AE: Affibody protein capture microarrays: synthesis and evaluation of random and directed immobilization of affibody molecules. Anal Biochem 341: 334-343, 2005.

28. Nordberg E, Orlova A, Friedman M, Tolmachev V, Stahl S, Nilsson FY, Glimelius B and Carlsson J: In vivo and in vitro uptake of ${ }^{111}$ In, delivered with the affibody molecule (ZEGFR:955)2, in EGFR expressing tumour cells. Oncol Rep 19: 853-857, 2008.

29. Vernet E, Konrad A, Lundberg E, Nygren PA and Graslund T: Affinity-based entrapment of the HER2 receptor in the endoplasmic reticulum using an affibody molecule. J Immunol Methods 338: 1-6, 2008.

30. Orlova A, Tolmachev V, Pehrson R, Lindborg M, Tran T, Sandstrom M, Nilsson FY, Wennborg A, Abrahmsen L and Feldwisch J: Synthetic affibody molecules: a novel class of affinity ligands for molecular imaging of HER2-expressing malignant tumors. Cancer Res 67: 2178-2186, 2007.

31. Wallberg $H$ and Orlova A: Slow internalization of anti-HER2 synthetic affibody monomer ${ }^{111}$ In-DOTA-ZHER2:342-pep2: implications for development of labeled tracers. Cancer Biother Radiopharm 23: 435-442, 2008.

32. Fan Z, Lu Y, Wu X and Mendelsohn J: Antibody-induced epidermal growth factor receptor dimerization mediates inhibition of autocrine proliferation of A431 squamous carcinoma cells. J Biol Chem 269: 27595-27602, 1994. 